\title{
LncRNA POU3F3 Promotes Cancer Cell Proliferation, Migration, and Invasion in Renal Cell Carcinoma by Downregulating LncRNA GAS5
}

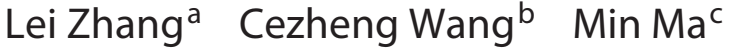 \\ aDepartment of Urology, The Second Hospital, Cheeloo College of Medicine, Shandong University,

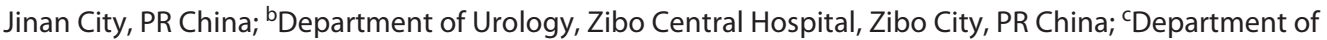 \\ Urology, Jinhua Central Hospital, Jinhua City, PR China
}

\section{Keywords}

Renal cell carcinoma · POU3F3 - GAS5

\begin{abstract}
Background: LncRNAs play regulatory roles in diverse nephrological disorders, including renal cancer. Overexpression of IncRNA POU3F3 (POU3F3) has only been reported in esophageal squamous-cell carcinomas, indicating POU3F3 may be an oncogene in this disease. LncRNA GAS5 (GAS5) was reported to be a suppressor in various tumors. However, the roles and underlying mechanism of POU3F3 and GAS5 involved in renal cell carcinoma (RCC) remain unknown. Methods: Real-time quantitative PCR and in situ hybridization were performed to determine the expression of POU3F3 and GAS5 in paired tumor and adjacent healthy tissues donated by 68 RCC patients. The prognostic values of POU3F3 and GAS5 for RCC were analyzed by performing a 5-year follow-up study. Overexpression of POU3F3 and GAS5 was achieved in RCC cells to explore the interactions between them. Transwell assay and cell proliferation assay were performed to evaluate the role of POU3F3 and GAS5 in regulating RCC cell proliferation, migration, and invasion. Results: In the present study, we found that POU3F3 was upregulated while GAS5 was downregulated in tumor tissues than that in adjacent healthy tissues of patients with RCC. In situ
\end{abstract}

karger@karger.com www.karger.com/kbr

Karger $\stackrel{\text { ' }}{5}$

GOPEN ACCESS
(C) 2021 The Author(s)

Published by S. Karger AG, Basel

This article is licensed under the Creative Commons AttributionNonCommercial-NoDerivatives 4.0 International License (CC BYNC-ND) (http://www.karger.com/Services/OpenAccessLicense) Usage and distribution for commercial purposes as well as any distribution of modified material requires written permission. hybridization analysis showed that POU3F3 was mostly expressed in tumor tissues, while GAS5 was mostly expressed in adjacent healthy tissues. High level of POU3F3 and low level of GAS5 were closely correlated with poor prognosis of RCC patients. Expression levels of POU3F3 and GAS5 were significantly and inversely correlated in tumor tissues but not in adjacent healthy tissues of RCC patients. Overexpression of POU3F3 mediated the downregulation of GAS5 in RCC cells, while GAS5 overexpression failed to significantly affect POU3F3 expression. Overexpression of POU3F3 led to promoted, while GAS5 overexpression led to inhibited proliferation, migration, and invasion of RCC cells. In addition, GAS5 overexpression attenuated the enhancing effects of POU3F3 overexpression on cancer cell proliferation, migration, and invasion. Conclusion: POU3F3 promoted cell proliferation, migration, and invasion in RCC possibly by downregulating GAS5.

(C) 2021 The Author(s)

Published by S. Karger AG, Basel

\section{Introduction}

Renal cell carcinoma (RCC) is a type of cancer developed from the renal epithelium and accounts for $>90 \%$ cases of all cancers in the kidney [1]. RCC accounts for about $3 \%$ of all malignancies [2]. In the USA, RCC affects 
$>60,000$ new cases and causes $>14,000$ deaths every year [3]. In spite of efforts made on the treatment of RCC, survival of RCC patients is still poor, partially due to the fact that a considerable portion of patients with RCC are diagnosed at advanced stages, and the prevalence of tumor metastasis is high by the time of the first diagnosis [4]. At present, overall survival rate of RCC is still low even after active treatment [5].

Long noncoding RNAs (lncRNAs) are a group of nonprotein RNA transcripts composed of $>200$ nucleotides [6]. Different from protein-coding messenger RNAs, lncRNAs participate in physiological and pathological processes through the form of RNA [7]. A growing body of literature has shown that lncRNAs are key players in diverse nephrological disorders, such as kidney injuries and renal cancers [8-11]. Overexpression of POU3F3 has only been reported in esophageal squamous-cell carcinomas, indicating POU3F3 may be an oncogenic lncRNA in this disease [12]. GAS5 plays a role as a tumor suppressor in RCC [13]. Our preliminary transcriptome analysis showed that POU3F3 and GAS5 expression levels were inversely correlated in RCC cells. In the present study, we showed that POU3F3 promoted cell proliferation, migration, and invasion in RCC possibly by downregulating GAS5.

\section{Materials and Methods}

\section{Subjects, Specimens, and Cell Lines}

Our study included 68 patients with RCC (clear cell RCC) who were diagnosed and treated in the second hospital of Shandong University from January 2011 to May 2013. Inclusion criteria: (1) patients diagnosed by pathological tests, (2) patients who completed treatment and 5-year follow-up, and (3) patients with complete medical record. Exclusion criteria: (1) patients who were complicated with other diseases, (2) patients transferred to other hospitals during treatment, and (3) patients who died of other causes during follow-up. Tumor tissue and adjacent healthy tissues were collected from each patient. All tissues were confirmed by 3 experienced pathologists. According to AJCC staging, there were 12 cases at stage I, 18 cases at stage II, 15 cases at stage III, and 23 cases at stage IV. All patients signed informed consent, and this study passed the review of the Ethics Committee of the second hospital of Shandong University.

Human RCC cell line Caki-1 was used in this study to perform all in vitro experiments. Cells were purchased from American Type Culture Collection (ATCC, Manassas, VA, USA). Cells were cultivated in ATCC-formulated McCoy's 5a Medium containing $10 \% \mathrm{FBS}$ at $37^{\circ} \mathrm{C}$ in a $5 \% \mathrm{CO}_{2}$ incubator.

\section{Real-Time Quantitative PCR}

To detect the expression of POU3F3 and GAS5, total RNA was extracted using Trizol reagent (Invitrogen, Waltham, MA, USA), reverse transcription was performed using Applied Biosystems ${ }^{\mathrm{TM}}$ High-Capacity cDNA Reverse Transcription Kit, and PCR reaction systems were prepared using SYBR ${ }^{\circledR}$ Green Real-Time PCR Master Mixes (Thermo Fisher Scientific, Waltham, MA, USA). Primers of POU3F3, GAS5, and endogenous control were designed and synthesized by Sangon (Shanghai, China). Expression levels of POU3F3 and GAS5 were normalized to GAPDH according to the $2^{-\triangle \Delta C T}$ method.

\section{Cell Transfections}

POU3F3 and GAS5 expression vectors and empty vectors were designed and constructed by Sangon (Shanghai, China). Caki-1 cells were cultivated overnight to reach $70-90 \%$ confluence. Lipofectamine 2000 reagent (Thermo Fisher Scientific, Inc.) was used to perform all cell transfections with vectors at a dose of $10 \mathrm{nM}$, and all operations were performed according to manufacturer's instruction. Treatment with Lipofectamine 2000 reagent only was the control group. Treatment with empty vectors was the negative control (NC) group.

\section{Cell Proliferation Assay}

Expression of POU3F3 and GAS5 was detected by RT-qPCR at $24 \mathrm{~h}$ after transfection. Cells were harvested to perform cell proliferation assay in cases of POU3F3 and GAS5 overexpression rates above $200 \%$ compared with the control group. In brief, cells were used to prepare single-cell suspensions, and cell density was adjusted to $3 \times 10^{4}$ cells per mL. $0.1 \mathrm{~mL}$ cell suspension containing 3 $\times 10^{3}$ cells was added into each well of a 96-well plate. Cells were cultivated at $37^{\circ} \mathrm{C}$ in a $5 \% \mathrm{CO}_{2}$ incubator, and $10 \mu \mathrm{L}$ CCK-8 solution was added into each well 24, 48, 72, and $96 \mathrm{~h}$ later. After that, cells were cultivated for additional $4 \mathrm{~h}$, and optical density at 450 $\mathrm{nm}$ was measured.

\section{Transwell Migration and Invasion Assay}

Expression of POU3F3 and GAS5 was detected by RT-qPCR at $24 \mathrm{~h}$ after transfection. Cells were harvested to perform cell migration and invasion assay in cases of POU3F3 and GAS5 overexpression rates above $200 \%$ compared with the control group. In brief, cells were used to prepare single-cell suspensions using serum-free cell culture medium, and cell density was adjusted to $3 \times 10^{4}$ cells per mL. $0.1 \mathrm{~mL}$ cell suspension containing $3 \times 10^{3}$ cells was added into the upper chamber, while the lower chamber was filled with cell culture medium containing $20 \%$ FBS. Cells were cultivated at $37^{\circ} \mathrm{C}$ in a $5 \% \mathrm{CO}_{2}$ incubator for $2 \mathrm{~h}$. The upper chamber membrane was then stained with $0.5 \%$ crystal violet (Sigma-Aldrich, St. Louis, MO, USA) at room temperature for $20 \mathrm{~min}$. Migration and invasion assays were performed in the same way except that the upper chamber was precoated with Matrigel (356234; Millipore, Burlington, MA, USA) before invasion assay. Stained cells were counted under an optical microscope.

\section{In situ Hybridization}

Based on the manufacturer's recommendations (Qiagen, 339450) and the method demonstrated in the research of Gasch et al. [14], an optimized in situ hybridization protocol was applied. In brief, 8- $\mu \mathrm{m}$ tissue sections were deparaffinized in $x y-$ lene, washed, and rehydrated in serial dilution of ethanol $/ \mathrm{dH}_{2} \mathrm{O}$. Antigen retrieval was performed in citrate buffer $\mathrm{pH} 6.0$ at $100^{\circ} \mathrm{C}$. 
Fig. 1. POU3F3 and GAS5 expression levels were altered in tumor tissues of RCC patients. RT-qPCR results showed that POU3F3 was significantly upregulated $(\mathbf{A})$, while GAS5 (B) was significantly downregulated in tumor tissues than that in adjacent healthy tissues. In situ hybridization analysis showed that POU3F3 was mostly expressed in tumor tissues (C), while GAS5 (D) was mostly expressed in adjacent healthy tissues. Scale bar, $25 \mu \mathrm{m}$. ${ }^{*} p<0.05$. RCC, renal cell carcinoma.

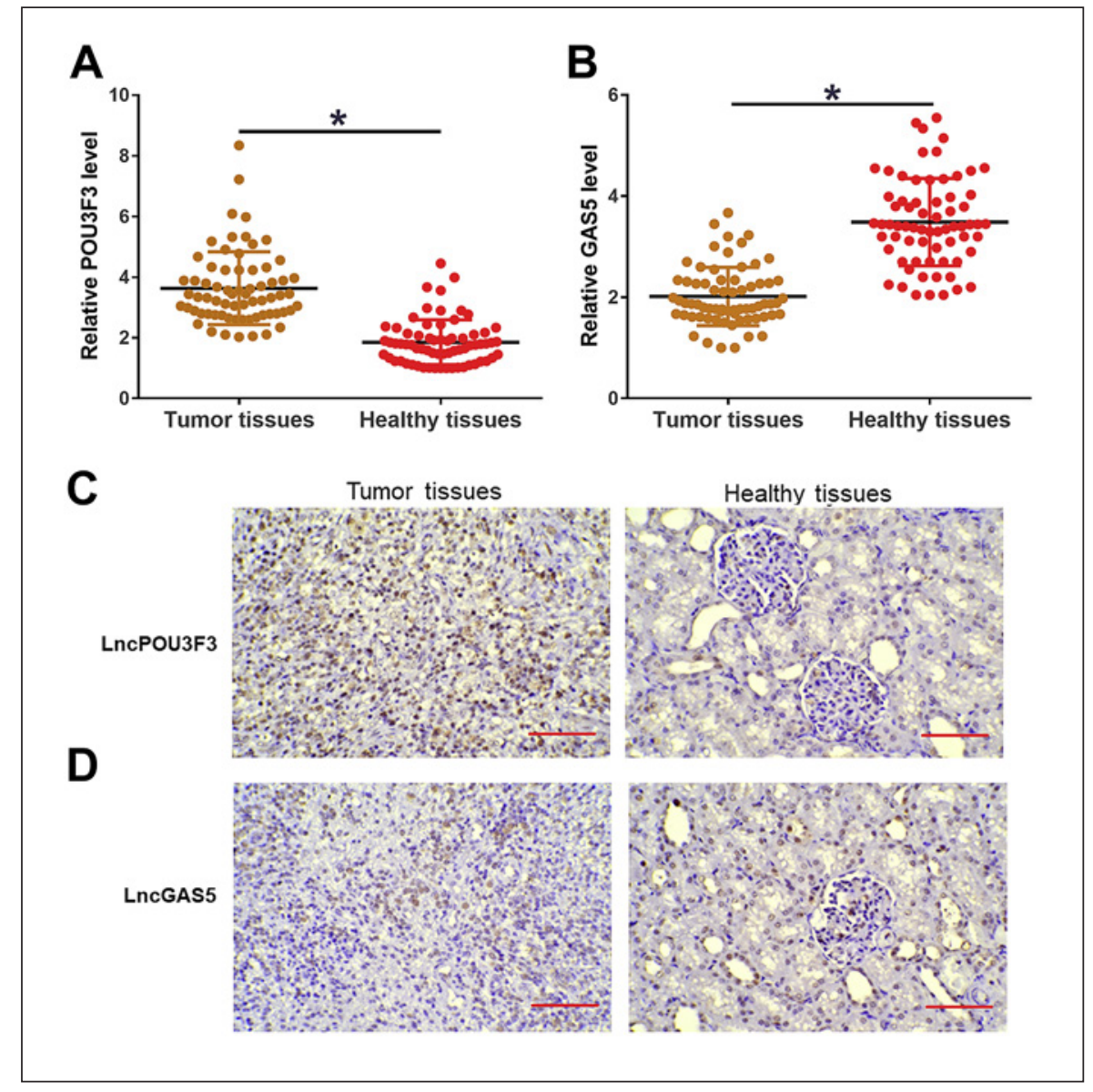

\section{Statistical Analysis}

Experiments were performed in a triplicate manner, and data were recorded as mean \pm standard deviation. All statistical analyses were performed using GraphPad Prism 6 software. Comparisons of expression levels of POU3F3 and GAS5 between tumor tissues and adjacent healthy tissues were performed by the paired $t$ test. Comparisons among multiple groups were performed by one-way ANOVA and Tukey test. Correlations between expression levels of POU3F3 and GAS5 were analyzed by the Pearson correlation coefficient. According to Youden's index, patients were divided into high $(n=31)$ and low $(n=37)$ POU3F3 groups and high $(n=30)$ and low $(n=38)$ GAS5 groups. Survival curves were plotted using the Kaplan-Meier method for each group and compared by the log-rank test. $p<0.05$ was considered to be statistically significant.

\section{Results}

POU3F3 and GAS5 Expression Levels Were Altered in Tumor Tissues of RCC Patients

Expression of POU3F3 and GAS5 in tumor tissues and adjacent healthy tissues of 68 patients with RCC was de- tected by RT-qPCR. As shown in Figure 1A, POU3F3 was significantly upregulated (Fig. 1A), while GAS5 (Fig. 1B) was significantly downregulated in tumor tissues than that in adjacent healthy tissues $(p<0.05)$, which was inconsistent with the result of in situ hybridization assay (Fig. 1C, D). In situ hybridization analysis showed that POU3F3 was mostly expressed in tumor tissues (Fig. 1C), while GAS5 was mostly expressed in adjacent healthy tissues (Fig. 1D).

\section{High Level of POU3F3 and Low Level of GAS5 Were Closely Correlated with Poor Prognosis of RCC Patients}

According to Youden's index, patients were divided into high $(n=31)$ and low $(n=37)$ POU3F3 groups and high $(n=30)$ and low $(n=38)$ GAS5 groups. As shown in Figure 2, patients with higher level of POU3F3 (Fig. 2A) and lower level of GAS5 (Fig. 2B) showed significantly lower overall survival rates. 
Fig. 2. High level of POU3F3 and low level of GAS5 were closely correlated with poor prognosis of RCC patients. Survival curve analysis showed that high level of POU3F3 (A) and low level of GAS5 (B) were closely correlated with poor prognosis of RCC patients. RCC, renal cell carcinoma.

Fig. 3. Expression levels of POU3F3 and GAS5 were significantly and inversely correlated in tumor tissues. The Pearson correlation coefficient showed that expression levels of POU3F3 and GAS5 were significantly and inversely correlated in tumor tissues (A), but not in adjacent healthy tissues $(\mathbf{B})$.

Fig. 4. Overexpression of POU3F3 mediated the downregulation of GAS5 (A), while GAS5 overexpression failed to significantly affect POU3F3 expression (B). ${ }^{*} p<$ 0.05 .
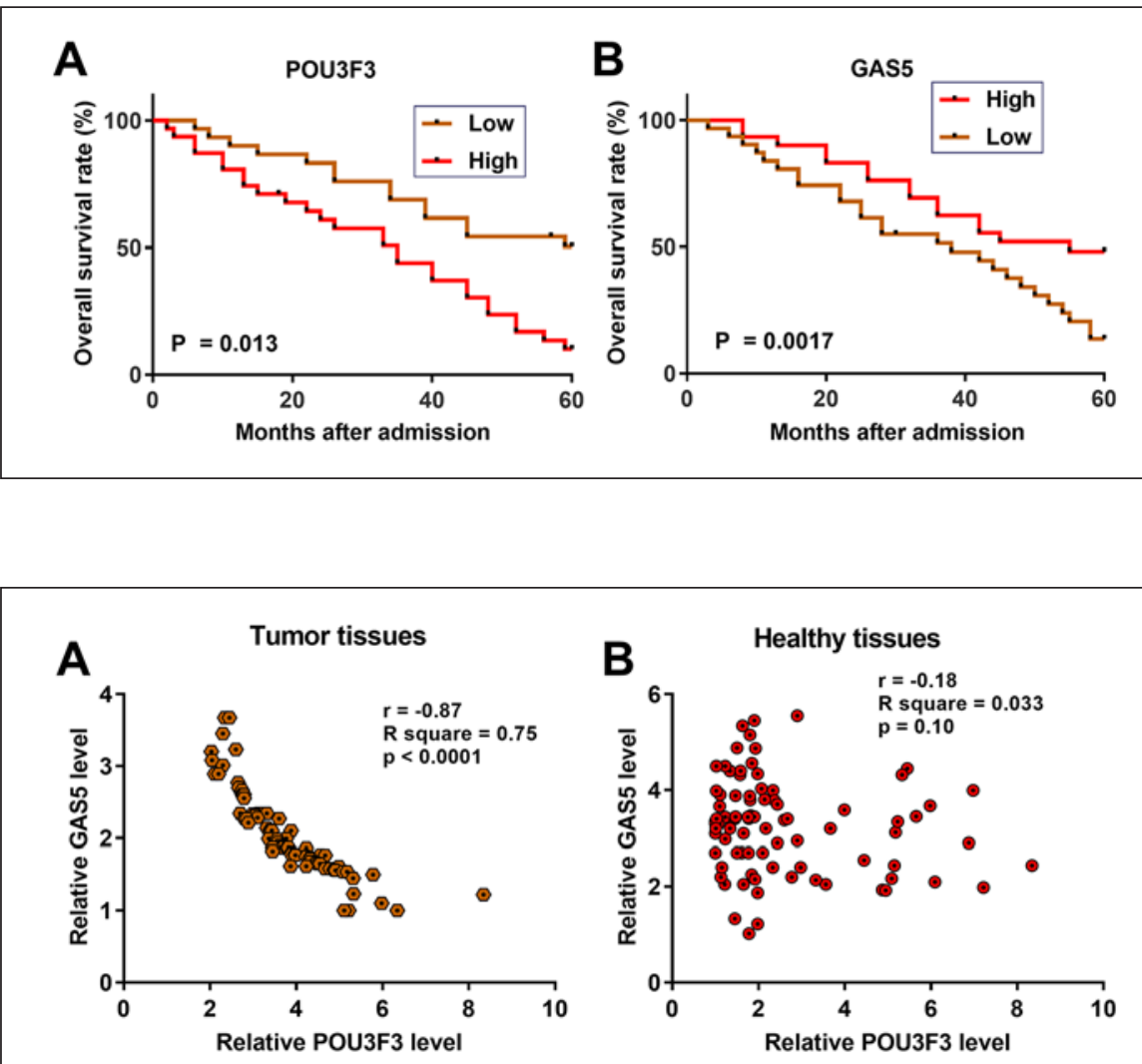
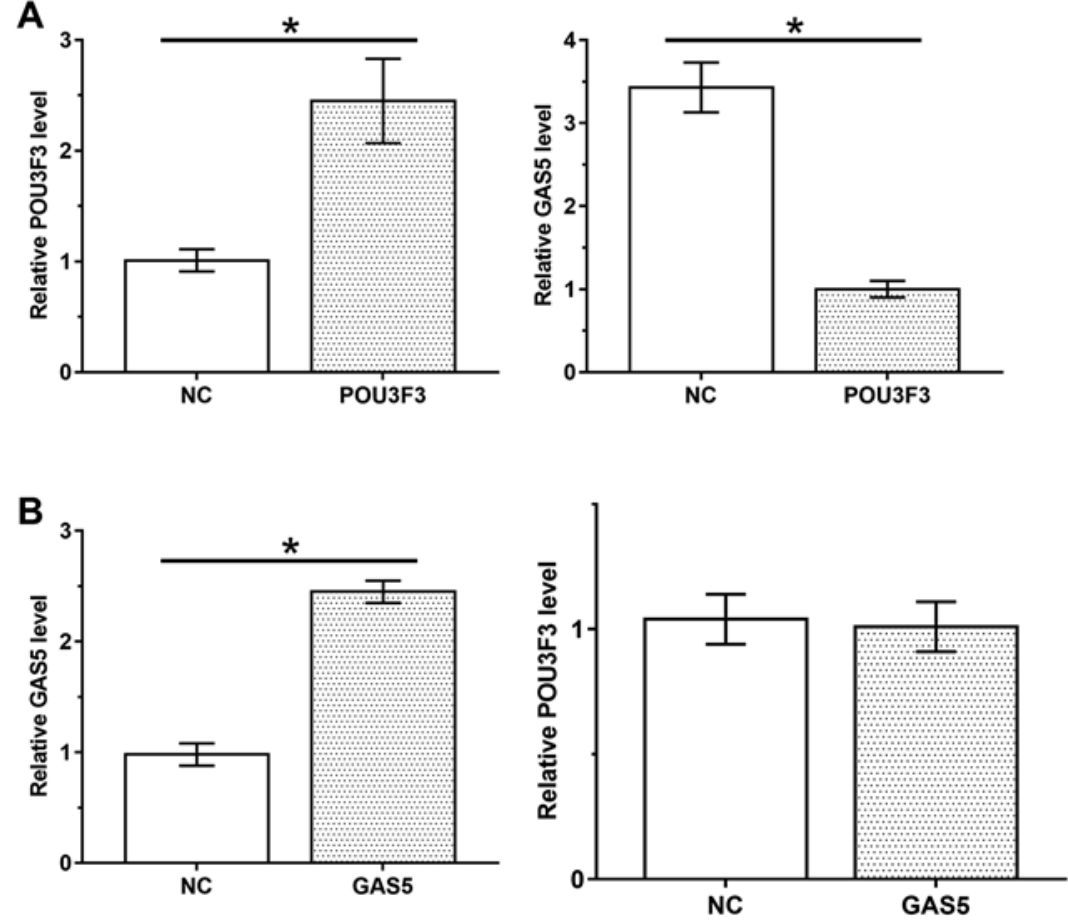


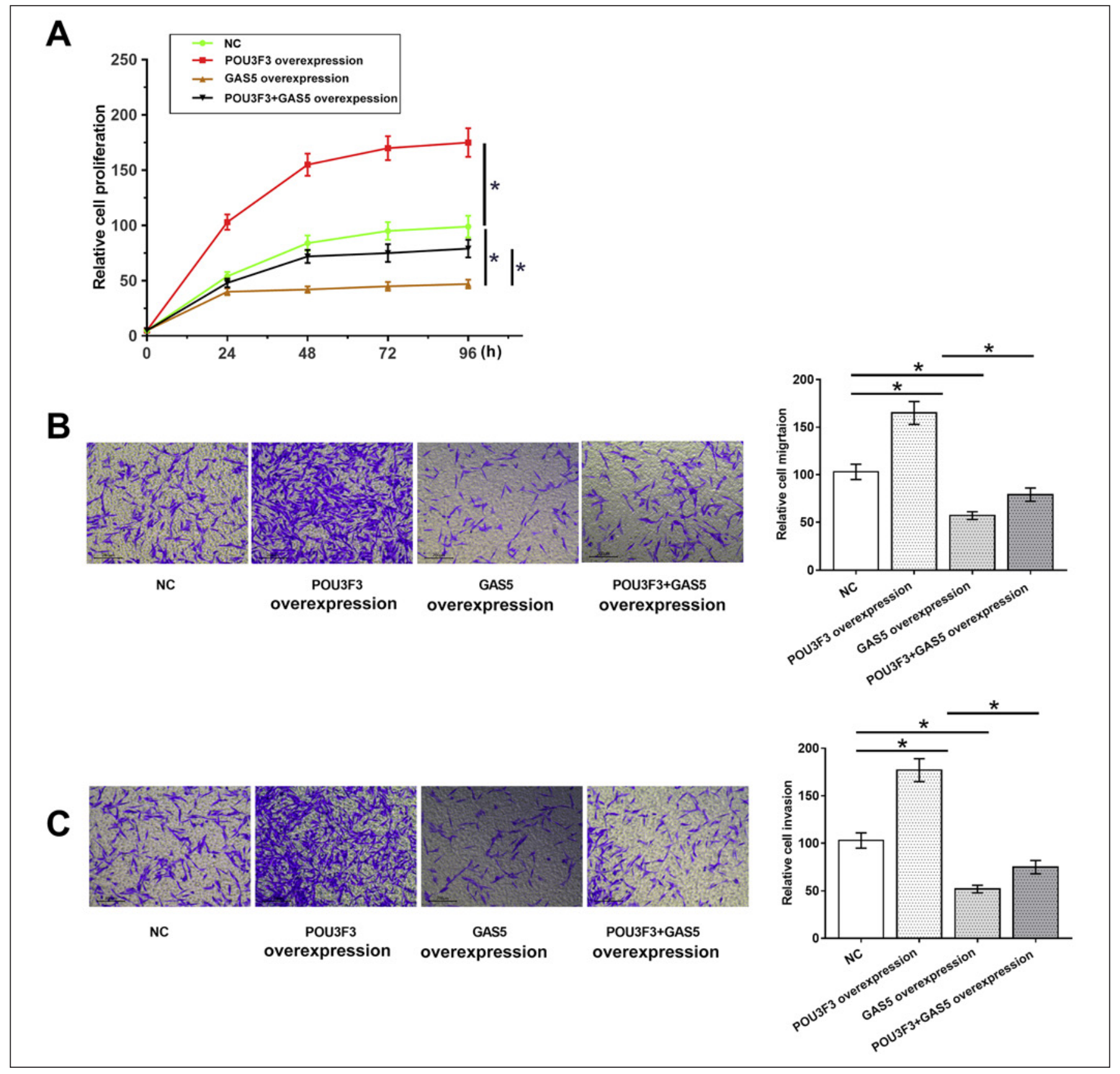

Fig. 5. POU3F3 regulates RCC cell behaviors through GAS5. Overexpression of POU3F3 led to promoted, while GAS5 overexpression led to inhibited proliferation (A), migration (B), and invasion (C) of RCC cells. In addition, GAS5 overexpression attenuated the enhancing effects of POU3F3 overexpression on cancer cell proliferation, migration, and invasion. ${ }^{*} p<0.05$. RCC, renal cell carcinoma.

Expression Levels of POU3F3 and GAS5 Were

Significantly and Inversely Correlated in Tumor

Tissues

Correlations between expression levels of POU3F3 and GAS5 were analyzed by the Pearson correlation coef- ficient. The results showed a significant and inverse correlation between expression levels of POU3F3 and GAS5 in tumor tissues (Fig. 3A). However, the correlation between expression levels of POU3F3 and GAS5 was not significant in adjacent healthy tissues (Fig. 3B). 
Overexpression of POU3F3 Mediated the

Downregulation of GAS5

POU3F3 and GAS5 were overexpressed in human RCC cell line Caki-1 to further investigate the interactions between POU3F3 and GAS5. Compared with NC groups, overexpression of POU3F3 mediated the downregulation of GAS5 (Fig. 4A, $p<0.05$ ), while GAS5 overexpression failed to significantly affect POU3F3 expression (Fig. 4B, $p<0.05$ ).

POU3F3 Regulates RCC Cell Behaviors through GAS5 Compared with NCgroups, overexpression of POU3F3 led to promoted, while GAS5 overexpression led to inhibited proliferation (Fig. 5A), migration (Fig. 5B), and invasion (Fig. 5C) of RCC cells $(p<0.05)$. In addition, GAS5 overexpression attenuated the enhancing effects of POU3F3 overexpression on cancer cell proliferation, migration, and invasion.

\section{Discussion}

The expression pattern of POU3F3 has only been characterized in esophageal squamous-cell carcinomas [12], while its role in this disease and its involvement in other human diseases are still unknown. The key finding of the present study is that POU3F3 was upregulated in RCC and might promote RCC by downregulating GAS5.

Downregulation of GAS5 is frequently observed during the development of different types of cancers [15-18]. Decreased expression of GAS5 led to the accelerated cancer development and predicted poor prognosis [15-17]. Besides that, inhibition of GAS5 also led to develop chemoresistance during chemotherapy. Many studies have demonstrated the GAS5 functions as a tumor suppresser that promotes cell death and cellular growth inhibition of cancer cells through regulation of cell cycle and cell apoptosis [19-21]. In a recent study, it was reported that GAS5 was downregulated in RCC, and the overexpression of GAS5 inhibited cancer development by inhibiting cancer cell proliferation, migration, and invasion. Consistent results were observed in the present study. Besides that, our follow-up study also suggests that downregulation of GAS5 is closely correlated with the poor survival of RCC patients, indicating the potential role of GAS5 in RCC.

POU3F3 has been reported in various tumors, functioning as a cancer promoter. In nasopharyngeal carcinoma, POU3F3 promoted cancer cell migration and invasion by upregulating TGF- $\beta 1$ [22]. In prostate carcinoma, POU3F3 promoted cancer cell proliferation by upregulat- ing rho-associated protein kinase 1 [23]. In addition, POU3F3 promoted proliferation and inhibited apoptosis of triple-negative breast cancer cells by inactivating caspase 9 [24]. In the present study, we showed that POU3F3 was also upregulated in RCC. LncRNAs participate in human cancer through the interactions with proteins or even other noncoding RNAs, such as microRNAs [25-29]. However, based on our best knowledge, reports on the interactions between lncRNAs are rare [28]. In the present study, we showed that POU3F3 was likely an upstream inhibitor of GAS5 in RCC cells, and the inhibition of GAS5 by POU3F3 was involved in the regulation of the proliferation, migration, and invasion of RCC cells. In another study, Zhang et al. [29] reported that the expression of GAS5 was negatively regulated in miRNA-21. Therefore, the expression of GAS5 is regulated by multiple noncoding RNAs. Our study provided new insights in the pathogenesis of RCC. In conclusion, POU3F3 was upregulated in RCC and promoted RCC by downregulating GAS5.

\section{Statement of Ethics}

All patients signed informed consent, and this study passed the review of the Ethics Committee of the second hospital of Shandong University.

\section{Conflict of Interest Statement}

The authors declare that they have no conflicts of interest.

\section{Funding Sources}

The authors did not receive any funding.

\section{Author Contributions}

L.Z. designed and carried out the study. L.Z., C.Z.W., and M.M. participated in experiments and statistical analysis. L.Z. wrote the manuscript. All authors read and approved the final manuscript.

\section{References}

1 Hsieh JJ, Purdue MP, Signoretti S, Swanton C, Albiges L, Schmidinger M, et al. Renal cell carcinoma. Nat Rev Dis Primers. 2017;3: 17009.

2 Zhang WB, Pan ZQ, Yang QS, Zheng XM Tumor suppressive miR-509-5p contributes to cell migration, proliferation and antiapoptosis in renal cell carcinoma. Ir J Med Sci. 2013;182:621-7. 
3 Siegel R, Naishadham D, Jemal A. Cancer statistics, 2013. CA Cancer J Clin. 2013;63:1130.

4 Flanigan RC, Campbell SC, Clark JI, Picken MM. Metastatic renal cell carcinoma. Curr Treat Options Oncol. 2003;4(5):385-90.

5 Motzer RJ, Hutson TE, Tomczak P, Michaelson MD, Bukowski RM, Oudard S, et al. Overall survival and updated results for sunitinib compared with interferon alfa in patients with metastatic renal cell carcinoma. J Clin Oncol. 2009;27(22):3584-90.

6 Mercer TR, Dinger ME, Mattick JS. Long non-coding RNAs: insights into functions[J]. Nat Rev Genet. 2009;10(3):155-9.

7 Fatica A, Bozzoni I. Long non-coding RNAs: new players in cell differentiation and development[J]. Nat Rev Genet. 2014;15(1): 7-21.

8 Gutschner T, Diederichs S. The hallmarks of cancer: a long non-coding RNA point of view[J]. RNA Biol. 2012;9(6):703-19.

9 Zhu Y, Wei SW, Ding A, Zhu WP, Mai MF, Cui TX, et al. The long noncoding RNA ANRIL promotes cell apoptosis in lipopolysaccharide-induced acute kidney injury mediated by the TLR4/nuclear factor-kappa B pathway. Kidney Blood Press Res. 2020;45(2): 209-21.

10 Duan ZY, Cai G, Li JJ, Bu R, Chen XM, Urinary erythrocyte-derived miRNAs: emerging role in IgA nephropathy[J]. Kidney Blood Press Res. 2017;42(4):738-48.

11 Zhou P, Chen Z, Zou Y, Wan X. Roles of noncoding RNAs in acute kidney injury. Kidney Blood Press Res. 2016;41(6):757-69.

12 Tong YS, Wang XW, Zhou XL, Liu ZH, Yang TX, Shi WH, et al. Identification of the long non-coding RNA POU3F3 in plasma as a novel biomarker for diagnosis of esophageal squamous cell carcinoma. Mol Cancer. 2015; 14(1):3.
13 Qiao HP, Gao WS, Huo JX, Yang ZS. Long non-coding RNA GAS5 functions as a tumor suppressor in renal cell carcinoma. Asian Pac J Cancer Prev. 2013;14(2):1077-82.

14 Ma L, Reinhardt F, Pan E, Soutschek J, Bhat B, Marcusson EG, et al. Therapeutic silencing of miR-10b inhibits metastasis in a mouse mammary tumor model. Nat Biotechnol. 2010;28(4):341-7.

15 Cao S, Liu W, Li F, Zhao W, Qin C. Decreased expression of lncRNA GAS5 predicts a poor prognosis in cervical cancer. Int J Clin Exp Pathol. 2014;7(10):6776-83.

$16 \mathrm{Tu}$ ZQ, Li RJ, Mei JZ, Li XH. Down-regulation of long non-coding RNA GAS5 is associated with the prognosis of hepatocellular carcinoma. Int J Clin Exp Pathol. 2014;7(7):4303-9.

17 Tao R, Hu S, Wang S, Zhou X, Zhang Q, Wang $C$, et al. Association between indel polymorphism in the promoter region of $\ln$ cRNA GAS5 and the risk of hepatocellular carcinoma. Carcinogenesis. 2015;36(10): 1136-43.

18 Li W, Zhai L, Wang H, Liu C, Zhang J, Chen W, et al. Downregulation of LncRNA GAS5 causes trastuzumab resistance in breast cancer. Oncotarget. 2016;7(19):27778-86.

19 Liu Y, Zhao J, Zhang W, Gan J, Hu C, Huang $\mathrm{G}$, et al. lncRNA GAS5 enhances G1 cell cycle arrest via binding to YBX1 to regulate $\mathrm{p} 21 \mathrm{ex}$ pression in stomach cancer. Sci Rep. 2015;5: 10159.

20 Zhang Y, Luo R, Li X, et al. Long non-coding RNA GAS5 regulates the growth and metastasis of human cervical cancer cells via induction of apoptosis and cell cycle arrest. Arch Biochem Biophys. 2020;684:108320.
21 Chen D, Guo Y, Chen Y, Guo Q, Chen J, Li Y, et al. LncRNA growth arrest-specific transcript 5 targets miR-21 gene and regulates bladder cancer cell proliferation and apoptosis through PTEN. Cancer Med. 2019;00:113.

22 Li W, Wu X, She W. LncRNA POU3F3 promotes cancer cell migration and invasion in nasopharyngeal carcinoma by up-regulating TGF-ß1.BiosciRep.2019;39(1):BSR20181632.

23 Wan X, Xiang J, Zhang Q, Bian C. Long noncoding RNA POU3F3 promotes cancer cell proliferation in prostate carcinoma by upregulating rho-associated protein kinase 1. J Cell Biochem. 2018:1-6.

24 Yang J, Meng X, Yu Y, Pan L, Zheng Q, Lin W. LncRNA POU3F3 promotes proliferation and inhibits apoptosis of cancer cells in triplenegative breast cancer by inactivating caspase 9. Biosci Biotechnol Biochem. 2019;83(6): 1117-23.

25 Xiang JF, Yin QF, Chen T, Zhang Y, Zhang $\mathrm{XO}, \mathrm{Wu} \mathrm{Z}$, et al. Human colorectal cancerspecific CCAT1-L lncRNA regulates longrange chromatin interactions at the MYC locus. Cell Res. 2014;24(5):513-31.

26 Ferre F, Colantoni A, Helmer-Citterich M. Revealing protein-lncRNA interaction[J]. Brief Bioinform. 2015;17(1):106-16.

27 Jalali S, Bhartiya D, Lalwani MK, Sivasubbu S, Scaria V. Systematic transcriptome wide analysis of lncRNA-miRNA interactions. PLoS One. 2013;8(2):e53823.

28 Goro T, Iwakiri J, Kameda T, Hamada M, Asai K. Comprehensive prediction of lncRNARNA interactions in human transcriptome. BMC Genomics. 2016;17(1):153-64.

29 Zhang Z, Zhu Z, Watabe K, Zhang X, Bai C, $\mathrm{Xu}$ M, et al. Negative regulation of lncRNA GAS5 by miR-21. Cell Death Differ. 2013; 20(11):1558-68. 